Historic, Archive Document

Do not assume content reflects current scientific knowledge, policies, or practices. 



\title{
SUIRIST GLADIOLUS GARDEIS
}

\author{
3806 Easton Blva., \\ Des Hoines, Iowa.
}

\section{SPRIITG CLEAII UP S.LP}

These bulbs are grown from SEATSA treated stock, are clean,

true to name, and machine graded.

Terms:- Cash with order.

Discount:- on orders of $\$ 5.00$ to $\$ 10.00,10 \%$

on orders of 10.00 to $25.00,15 \%$.

on orders of 25.00 and up, $20 \%$.

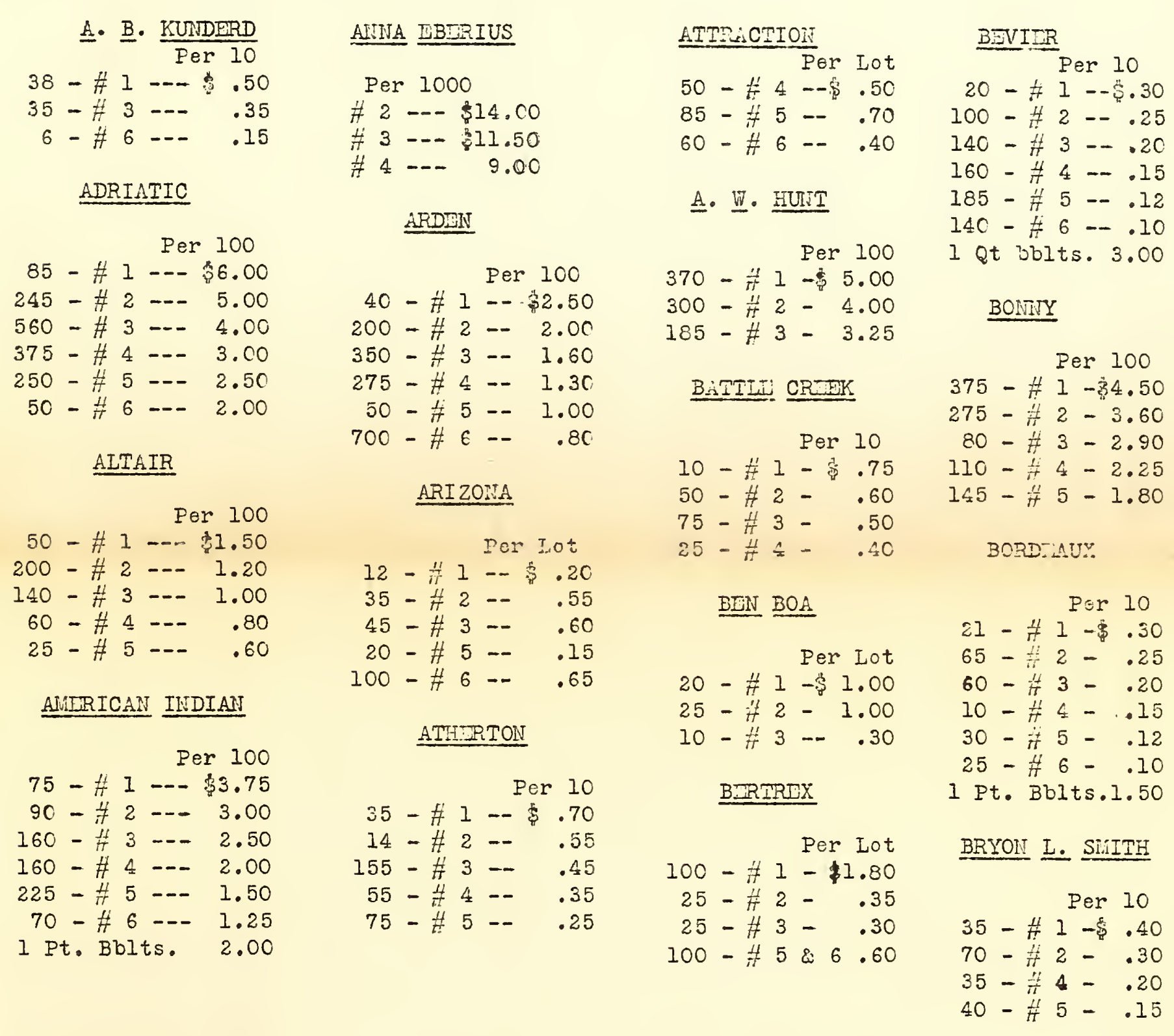



BRILLIANT

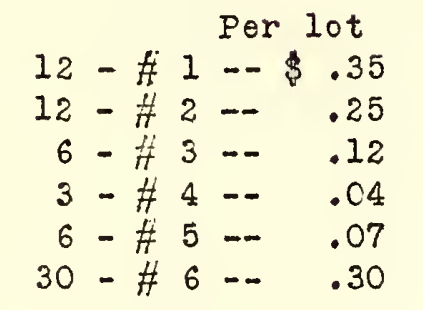

BUCKEYE

$$
\begin{array}{rrr} 
& \multicolumn{3}{c}{\text { Per } 10} \\
50-\# 1-\#-\$ & .80 \\
12-\# 2-- & .65 \\
7-\# 3-- & .55 \\
5-\# 4 & -. & .45 \\
15-\# 5-- & .35 \\
14-\# 6-- & .30 \\
\frac{1}{4} \text { Pt. Bblts. } & 1.00
\end{array}
$$

CAPELIA

$$
\begin{aligned}
& \text { Per } 100 \\
& 125 \text { - \#1 - } \$ 1.60 \\
& 85 \text { - \#2 - } 1.30 \\
& 200-\# 3-1.00 \\
& 65 \text { - \#4 - . } 80 \\
& 30-\# 5 \ldots .65
\end{aligned}
$$

\section{CARHEN SYLVA}

$190-\# 1 \quad \begin{array}{ll}\text { Per } 100 \\ -\# 4.00\end{array}$ $925-\# 2-3.20$ 1000 \# 3 -. 2.60

\begin{tabular}{|c|c|}
\hline AliDi ICAII & BEhUTY \\
\hline & $\begin{array}{l}\text { Por Lot } \\
-\$ 1.80\end{array}$ \\
\hline $15-\# 2$ & .70 \\
\hline $35-\frac{\pi}{\#} 3$ & 1.35 \\
\hline $10-\# 4$ & .30 \\
\hline
\end{tabular}

CINNAMON BEAR

\begin{tabular}{rrrr} 
& \multicolumn{2}{c}{ Per Lot } \\
$12-\#$ & 1 & -- & .50 \\
$18-\#$ & 2 &.- & .55 \\
$70-\#$ & 3 & -- & 1.80 \\
$20-\#$ & $4--$ & .40 \\
$15-\#$ & -4 & - & .25 \\
$10-\#$ & $6--$ & .15 \\
250 Bblts & & .20
\end{tabular}

\section{CIRCE}

Per 10

50 - \#1 - - . 40

$25-\# 2-.35$

15 - \# 3 - . 30

$5-\# 4-. \quad .25$

$10-\# 5 \quad-\quad .20$
E. I. SHAYIOR

Per 100

$$
\begin{array}{r}
200-\# 1-\# 2.80 \\
200-\# 2-2.25 \\
100-\# 3-1.70 \\
25-\# 4-1.35 \\
30-\# 5-1.00 \\
150-\# 6-.80
\end{array}
$$

DITNTR'S

DOROTHY UIIELER

Per 100

$50-\frac{H}{\#} 1-50$

$150-\# 2-1.25$

$135-\# 3-1.00$

$60-$ \# $4-.80$

$35-\# 5-.65$

DCROTHY MCKIBBIN

Per 10

$50-\# 2-\$ .20$

$160-\# 3-.16$

$90-\# 4-.13$

$15-\# 5-.10$

30 Bbits. .05

DR. ILKINS

Per 10

$85-\# 1-30$

$55-\# 2-.75$

$55-\# 3-.60$

DR. VAN FLETT

Per 10

$95-\# 1-\$ .40$

$70-\# 2-.35$

$65-$ H $3-.30$

$45-\# 4-.25$

65 - \#5 - .20

$50-\# 6-.15$
Per 100

$1300-\# 2-\$ 1.75$

$3200-\# 3-1.40$

$1900-\# 4-1.10$

$\underline{E L F}$

Per 100

$125-\# 1-\$ 4.00$

$220-\# 2-3.20$

$160-\# 3-2.50$

$65-\# 4-2.00$

$130-\# 5-1.50$

$375-\# 6-1.25$

1 Qt. Bblts. 4.00

EAIRIAND

Per 10

$15-\# 1-\$ .40$

$25-\# 2-.30$

$55-\not 33-.25$

$55-\# 4-.20$

$90-5 \& 6 \quad .15$

$500 \mathrm{Bblts}$.

.40

GIANT NYMPH

Per 100

$75-\# 2-\$ 2.80$

$400-\# 3-2.25$

$400-\# 4-1.80$

GLEITDAIE

Per 100

125 - \#I - 4.00

$450-\# 2-3.20$

$500-$ It $3-2.60$

$300-\# 4-2.00$

$125-\# 5-1.60$

GOLD DROP

Per 10

$15-\# 1-\$ .40$

$15-\# 2-.30$

$14-\# 2-.25$

$16-\# 4-.20$

$25-\# 5-.15$

$25-\# 6-.13$
GOLD

$$
\begin{aligned}
& \text { Per } 10 \\
& 25-\# 1-\$ .50 \\
& 90-\# 2-.40 \\
& 75-\# 3-.30 \\
& \text { GOLDIN MEASURS }
\end{aligned}
$$

\section{GOLDFN HEASURE}

$100-\# 1-\$ 4.00$

GOID THROAT

$$
\begin{aligned}
& \text { Per lot } \\
& 8-\# 1-\# .50 \\
& 5-\# 2-.25 \\
& 2-\# 3-.10 \\
& 4-\# 4-.15
\end{aligned}
$$

\section{GOSTEN}

Per $10 t$

$3-\# 1-\$ 20$

$12-\# 2-.55$

$4-\# 3-.15$

$10-\# 4-.30$

$65-\# 5-1.60$

\section{HOLLYHOCK}

$$
\begin{array}{r}
\text { Per } 10 \\
60-\# 1-\$ 1.50 \\
130-\# 2-1.20 \\
\text { IIDIAN MAID }
\end{array}
$$

$25-\# 1-\$ .80$

INDIAN SUMIER

$$
\begin{array}{r}
\text { Per } 10 \\
45-\# 1-\$ .60 \\
40-\# 2-.50 \\
60-\# 3-.40
\end{array}
$$

KASSON

$$
\begin{array}{r}
\text { Per } 100 \\
200-\# 1-\$ 5.00 \\
159-\# 2-4.00 \\
120-\# 3-3.00 \\
50-\# 4-\# 2.50 \\
30-\# 5-2.00 \\
25-\# 6-1.60
\end{array}
$$



KUIDIRD'S APPLE BLOSSOH

Per 10

$14-\# 1-\$ .65$

$23-\# 2-.55$

$45-\# 3-.45$

$20-\# 4-.35$

$20-\frac{\pi}{7} 56.25$

200 Bblts. .30

IA VANGE

Per 100

$100-\# 1-32.50$

$90-\# 2-2.00$

$25-\# 3-1.60$

$30-\# 4-1.25$

$145-\# 5-1.00$

$135-\# 6-.80$

1 Pt. Bblts. 1.50

IE NARECHAI FOCH

Per 100

$60-\# 1-\dot{3} 1.20$

$140-$ 莎 $2-1.00$

800 - $\frac{4}{i 5} 3-.80$

$280-$ t $4-.60$

IILY BLOTCH

Per 100

$5-\frac{4}{4} 1-30$

$64-$ 仕 $2-.25$

$115-\# 3-.20$

$240-\# 4-.16$

$30-\# 5-.12$

$300-\frac{10}{t} 6-.10$

$1 \frac{1}{2}$ Qts.Bblts 3.75

IILY VHIT:

Per 100

$275-\# 1-32.80$

$170-\# 2-1.45$

$100-\# 3-1.15$

$85-\not t 4-.90$

120 - 5 - .75

550 - \#6- . 60

1 Qt. Bblts. 2.00
LOVDLINESS

Per lot

$15-\# 1-.60$

$20-\# 2-.65$

$14-\# 3-.35$

$10-\# 5-.20$

$50-\# 6-.80$

100 Bblts. $\quad .10$

I:AIDIII'S BLUSH

Per 100

17 - \#1 - 1.50

$170-\# 2-1.20$

$255-\# 3-1.00$

$260-\# 4-.80$

$280-\# 5-.60$

$125-\# 6-.50$

1 Pts. Bblts.1.00

MA JESTIC

Por 10

$12-\# 2$ - .70

$35-\# 3-.55$

$25-\# 4-.45$

$10-\# 5-.35$

100 Bblts. .15

MAGIC

Per 10

$50-\frac{\# 1-.35}{\pi}$

$50-\# 2-.30$

$40-\# 4-.20$

25 if 5 \& $6 \quad .10$

\section{MARSTII IOCH}

Per 100

300 - 帮 1 - \$34.50

$1000-z^{2}-3.60$

825 - \#3-3.00

$330-\# 4-2.50$

\section{MARY PICKFORD}

Per 100

$450-\# 1-\$ 2.50$

$150-\frac{\| 1}{t} 2-2.00$

$115-\# 3-1.60$

$80-\# 4-1.30$

$2400-\# 5-1.00$

$1875-\# 6-.80$
MASTARPIECE

Per 10

$60-\# 1-\$ 1.00$

$145-\# 2-.80$

$95-43-.65$

MONA IISA

Per 100

$900-\# 1-\frac{\$ 1}{10} 80$

$800-\frac{41}{t} 2-1.50$

$650-\ddot{\# 3}-1.20$

$330-\# 4-.90$

$340-$ \# 5 - .75

NOTTLED BEUTY

Per Lot

$14-\# 1-\$ 1.40$

$10-\# 2-.80$

$15-\# 3-1.00$

MRS. A. Z. KUUTDLRD

\begin{tabular}{|c|c|c|c|}
\hline \multicolumn{4}{|c|}{ Per 10} \\
\hline 45 & - & $-\frac{n}{2}$ & .35 \\
\hline 40 & -42 & - & .30 \\
\hline 30 & $-\# 3$ & - & .25 \\
\hline 15 & $-\# 4$ & - & .20 \\
\hline 100 & $-H^{2} 5$ & - & .15 \\
\hline 100 & $-\frac{11}{\pi} 6$ & - & .12 \\
\hline
\end{tabular}

IRS. AGIIJS WHITITG

Per 100

60 - \#1 - 䧑1.50

$180-\frac{\pi}{\pi} 2-1.20$

$140-$ 莐 $3-.95$

$70-\ddot{t} 4-.75$

$25-\# 5-.60$

175 - 年 6 - .50

1 t pts. Bblts.

(2) \$.75 Per Pt.

DPS. F. FIIDLETON

$$
\begin{aligned}
& \text { Per Lot } \\
& 23-\# 1-\$ .45 \\
& 70-\# 2-1.30 \\
& 35-\# 3-.35 \\
& 40-\# 4-.35
\end{aligned}
$$

IMRS. H. E. BOTHIN

Per 1000

$$
\begin{array}{r}
1400-\# 1-\$ 22.00 \\
2240-\# 2-18.00 \\
1800-\# 3-14.50 \\
550-\# 4-11.50
\end{array}
$$

IRS. WM. KRIT

Per 100

$$
\begin{gathered}
160-\# 1-\$ 3.50 \\
75-\# 2-3.00 \\
38-\# 3-2.50 \\
23-\frac{\pi}{\pi} 4-2.00 \\
\text { MRS. MATT } \\
20-\# 2-\$ .70 \\
18-\frac{\#}{\pi} 3-.55 \\
6-\# 4-.15 \\
4-\frac{H}{\pi} 5-.20
\end{gathered}
$$

IIRS. RUDOLPH

SPRECKISS

$$
\begin{aligned}
& \text { Per } 100 \\
& 50-\# 1-52.50 \\
& 100-\text { 䓅2 - } 2.00 \\
& 525-\# 3-1.60 \\
& 400-\# 4-1.30 \\
& 250 \text { - \#5 - } 1.00 \\
& 60-\# 6-.80
\end{aligned}
$$

\section{NATALIS}

INDOGA

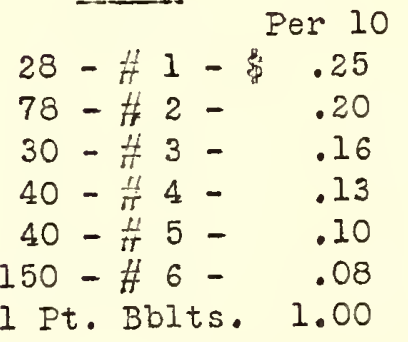




$$
\Gamma !
$$


NIAGARA

Per 100

$48-\frac{H}{\pi} 1-\$ 3.00$

$60-2-2.50$

$130-\# 3-2.00$

$255-\# 4-1.60$

$450-\# 5-1.25$

$215-\# 6-1.00$

1 Qt. Bblts. 3.50

\section{MOREIBEGA}

Per Lot

$5-\# 1-\$ .40$

$10-\# 2-.65$

$20-\# 3-1.05$

$15-\# 4-.60$

6 - \#5 - .20

NUBIAN

Per 10

$12-\frac{\pi}{11}-\$ .75$

$58-\# 2-.60$

$80-\# 3-.50$

$3.8-4-.40$

$25-\frac{46}{4} 6-.35$

NISP

Per 10
$165-\# 1 . \$ .40$
$100-\# 2-.35$
$53-\# 3-.30$
$28-\# c_{-}-.25$
$9-\# 5-.20$

1 P . Bults. 2.00

OLD SWOKY.

Per Lot

$1-\# 1-3.10$

$3-\# 2-.25$

$35-\frac{\#-2.00}{\#}$

$30-$ 形 $4-1.50$

$15-$ \# 5 - . 60

$50-\# 5-1.50$

100 Bblts. .25

\section{ONBGA}

Per Iot

$10-\# 2-\$ .50$

$16-\# 3-.65$

8 - \#4-.25

$4-\frac{H}{\#-.10}$
ORAIN

PEEP $O^{\prime} D A Y$

Per 100

$225-\# 1-\$ 5.00$

$180-\# 2-4.00$

$175-\# 3-3.20$

$147-\# 4-2.50$

$84-\# 5-2.00$

$40-\# 6-1.60$

2500 Bblts. 2.50

ORANGE BRILLIAINT

Per 10

$6-\# 1-\$ .35$

$30-\# 2-.30$

$30-\# 3-.25$

$78-\# 4-.20$

$50-\# 5-.16$

$115-\# 6-.13$

\section{ORANGE GIORY}

Per 100

$67-\# 1-\$ 3.00$

$120-\# 2-2.50$

$168-\# 3-2.00$

80 - \#4 - 1.60

$60-75-1,30$

$30-\# 6-1.00$

300 Bblts. .25

\section{PANAIIA}

80 Per 100

$133-\# 2-1.60$

$185 . . \# 3-1.30$

$85-\# 4-1.00$

25 - \#5-.80

2000 Bblts, 1.00

PESRE OF NASTH

$$
\text { per } 10
$$

$19-\# 1-\frac{1}{6} .70$

$80-\# 2-.60$

$165 \ldots 3 \ldots 50$

$115-\# 4.40$

100 - \#5-.20

$110-\# 6-.25$

$$
\begin{array}{r}
\text { Per } 10 \\
15-\# 1-\$ .50 \\
55-\# 2-.40 \\
25-\# 3-.35 \\
20-\# 4-.30 \\
50-\# 5-.25 \\
50-\# 6-.20 \\
1 \text { Pt. Bblts. } 3.00
\end{array}
$$

PINK BEAUTY BLOTCH

$$
\begin{array}{r}
\text { Per } 100 \\
875-\# 3-\# 1.75 \\
650-\# 4-1.40 \\
1300-\# 5-1.10 \\
\text { PIIK CLOUD }
\end{array}
$$

Per 10

$$
\begin{array}{r}
90-\# 1-\$ 2.50 \\
65-\# 2-2.00 \\
50-\# 3-1.60 \\
28-\# 4-1.30 \\
9-\# 5-1.00
\end{array}
$$

1 Pt. Bblts, 15.00

PINK IIIY

Per 1.00

140 - \#1-\$5.00

$570-\# 2-4.00$

$625-\frac{\| t}{\# t}-3.20$

$600-\# 4-2.60$

PINK WOIDER

Per 100

$100-\frac{t 1}{t 5}-\$ 2.25$

$130-\# 2-1,80$

$275-\# 3-1.50$

$600-\frac{11}{17}-1.20$

350 - 若5 - 1.00

$700-\# 6-.80$

PRIDE OE GOSIEN

Per 100

$115-$ H 1 - \$ 4.00

$100-\not 12-3.20$

$100-\# 3-2,60$

$45-\# 4-2.10$

$75-\# 5-1.70$

$70-\# 6-1.35$
PRIDE OF IAINCASTIR

Per 300

$190-\# 2-\$ 2,00$

$340-\# 3-1.60$

$225-\# 4-1.30$

$225-\# 5-1.00$

$170-\# 6-.80$

3 Pts. Bblts. 4.00

\section{PURPLE SPOT}

Per 100

$270-\# 1-\$ 4.00$

$140-\# 2-3.20$

$175-\# 3-2.60$

$105-\# 4-2.10$

$125-\# 5-1.70$

$100-\# 6-1.35$

\section{RARITY}

Per 100

$$
\begin{array}{r}
70-\# 1-\$ 5.00 \\
75-\# 2-\$ .00 \\
110-\# 3-3.20 \\
135-\# 4-2.60 \\
110-\# 5-2.10 \\
50-\# 6-1.70 \\
1000 \text { Bblts. } 1.00
\end{array}
$$

\section{ROSY GLOW}

Per 100

$50-\# 1-\$ 5,00$

180 - \#2-4.00

360 - 䓅 $3-3.20$

$60-\# 4-2.60$

1 Pt. Bblts. 2.50

ROSAIIND

$$
\begin{aligned}
& \text { Per } 10 \\
& 34-\# 1-\$ 3.00 \\
& 25-\# 2-\frac{H}{H} 2.50 \\
& 22-\# 3-2.00
\end{aligned}
$$

ROSE ASH

Per 100

$250-\# 3-\$ 2.50$

$325-\# 4-2.00$

$600-\frac{H}{1} 5-1.60$

$125-\# 6-1.25$ 

ROUGH TORCH

Per lot

$55-\# 1-\$ 2.20$

$110-\# 2-3.50$

$30-\# 3-.75$

$12-\# 4-.25$

$5-\# 5-.10$

500 Bblts. .40

RUFFLED AITRICA

Per 10

$15-\# 1-\$ .80$

$25-\# 2-.65$

$30-\# 3-.55$

$30-\# 4-.45$

RUFFLED BEAUTY

Per 10

$70-\# 1-\$ .80$

$110-\# 2-.65$

$155-\# 3-.55$

$135-\# 4-.45$

RICHARD DIJNER

Per 10

$25-\not 1 t 1-60$

$20-\# 2-.50$

$210-\# 3-.40$

$90-\frac{H}{t} 4-.35$

$25-\# 5-.30$

I Qt. Bblts. 8.00

SEBESTA

$110-\frac{H}{\# 2-\$ 4.00}$

275 - \#3-3.20

$400-\# 4-2.60$

$350-\# 5-2.00$

$500-\# 6-1.60$

SULPHUR FRILIS

Per 100

$300-\# 1-\$ 10.00$

$150-\# 2-8.00$

$135-\# 3-6.50$

$250-\# 4-5.25$
SCARLET PRIICEPS

Per 100

$38-\# 1-33.00$

$85-\# 2-2.40$

$40-\# 3-1.95$

$425-\# 4-1.65$

SULIIER BLOSSOM

Per 100

210 - \# I - \$3,50

$100-\# 2-3.00$

$100-\# 3-2.50$

$80-\# 4-2.00$

$100-\# 5-1.60$

I $\frac{1}{2}$ Pts. Bblts

2.75

SUMMER BEATY

Per 100

$33-\# 1-\$ 3.00$

$88-\# 2-2.40$

$85-\# 3-2.00$

$105-\# 4-1.60$

$125-\# 5-1.50$

$250-\# 6-2.50$

妾 Pt. Bblts. $\quad .75$

SMIIES

Per 100

$45-\# 1-\$ 7.00$

$65-\# 2-5.60$

$110-\# 3-4.50$

$70-\# 4-3.50$

$65-\# 5-2.80$

$100-\# 6-2.25$

300 Bblts. $\quad .60$

SIOW BOY

Per 100

$75-\# 1-\$ 4.50$

$150-\# 2-3.20$

$100-$ \#3 $3-2.60$

$160-\# 4-2.20$

$150-\# 5-1.80$
TEMPA

Per 100

165 - \#1 - \$6.00

$135-\# 2-5.00$

$95-\# 3-4.00$

1 Pt. Bblts. 3.00

THOIAAS $\mathrm{T} \cdot$ KENT

Per 100

$$
\begin{array}{r}
45-\# 1-\$ 4.00 \\
80-\# 2-3.20 \\
75-\# 3-2.50 \\
60-\# 4-2.00 \\
75-\# 5-1.60 \\
60-\# 6-1.30 \\
300 \text { Bblts. }
\end{array}
$$

THISTLE

$$
\begin{array}{r}
\text { Per } 10 \\
23-\# 1-\$ .60 \\
34-\# 2-\frac{11}{\# 1}-.50 \\
30-\# 3-.40 \\
30-\# 4-.35 \\
\text { ULYSSISS }
\end{array}
$$

Per 100

120 - \#1 - \$7.00

$95-\# 2-5.60$

$170-\# 3-4.50$

$110-\# 4-3.60$

VEITURA

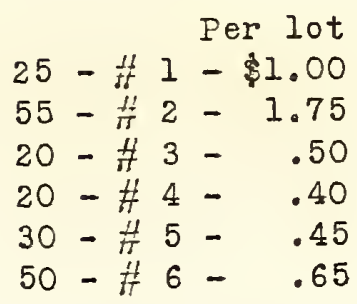

\section{VIOLET BISAUTY}

Per 100

$135-\# 1-\$ 2.50$

$400-\# 2-2.00$

$295-\# 3-1.60$

$100-\# 4-1.25$
WATERISELON

Per Lot

$$
\begin{array}{r}
3-\# 1-\# .30 \\
6-\# 2-.50 \\
18-\# 3-1.20 \\
6-\# 4-.35 \\
4-\# 5-.20
\end{array}
$$

WHITE DELIGET

Per 100

$190-\frac{\#-\$ 3.00}{\hbar t} 1-\$ 300$

$150-\# 2-2.40$

$75-\# 3-2.00$

$64-\# 4-1,50$

WHITE IVORY

Per 100

$40-\# 1-\$ 4.00$

$100-\# 2-3.20$

$110-\# 3-2.60$

$110-\# 4-2.00$

$75-\# 5-1.60$

WHITE GIANT

Per 10

$65-\# 1-\$ .40$

$60-\# 2-.35$

$25-\# 3-.30$

$26-\# 4-.25$

YOUELI'S FAVORITE

Per 100

$110-\# 1-\$ 3.50$

$300-\# 2-2.80$

$200-\# 3-2.25$

$100-\# 4-1.80$

$125-\# 5-1.40$ 
a

$\therefore \therefore$

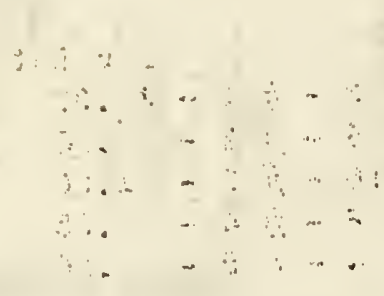

a
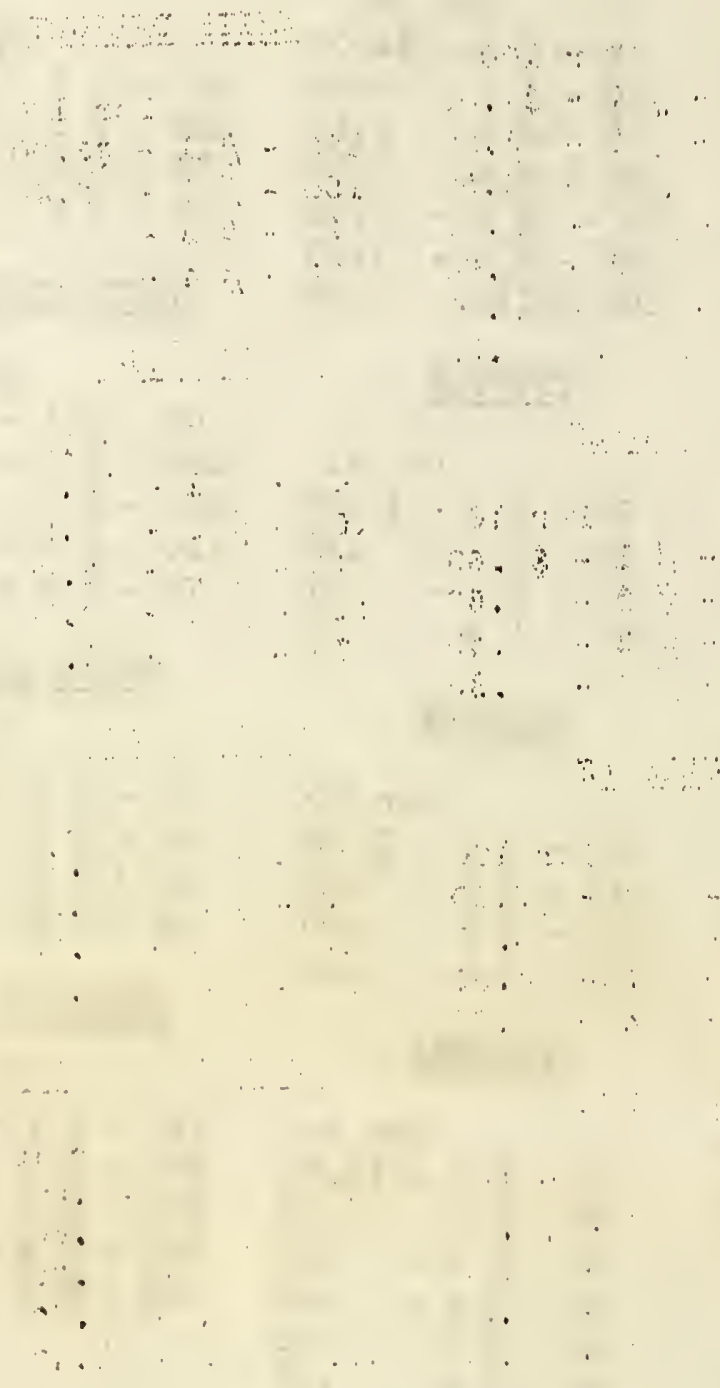

..

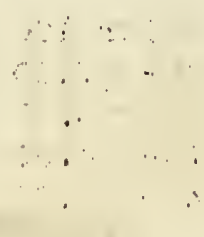

\section{$\therefore \quad$}

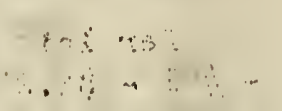

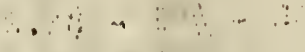

$\begin{array}{llll} & \cdots & \cdots & \cdots \\ \ddots & \cdots & \vdots\end{array}$

$\begin{array}{lll}\therefore & - \\ \vdots & - \\ \therefore & \end{array}$

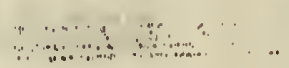
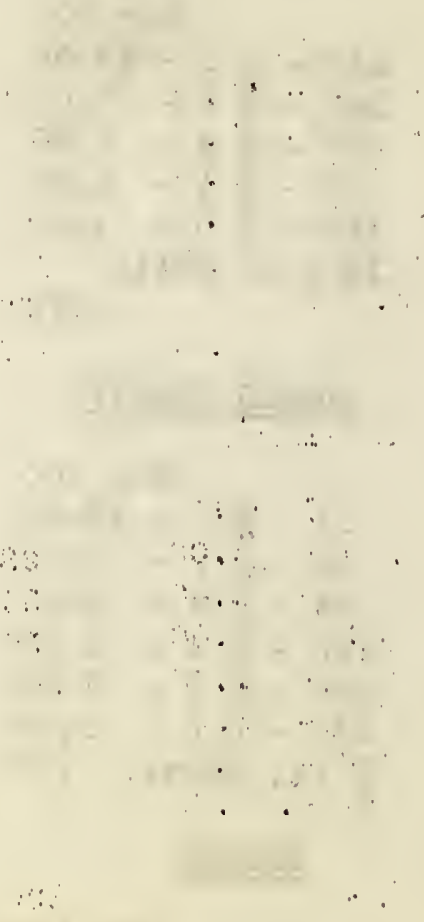